\title{
Dosimetric comparison of 3DCRT versus IMRT in whole breast irradiation of early stage breast cancer
}

\author{
Mudasir Ashraf ${ }^{1,3}$, Nandigam Janardhan², Perumal Bhavani'2, Radhakrishana Shivakumar', \\ Syed Ibrahim ${ }^{2}$, Palreddy Yadagiri Reddy ${ }^{3}$, Jagdessan Surrendharen², Balakrishna Sarangnathan², \\ Ben Johnson'2, Bhagavathula Madhuri², Rayees Ahmad Dar ${ }^{4}$ \\ ${ }^{I}$ Department of Radiological Physics and Bio-Engineering, Sher-i-Kashmir Institute of Medical Sciences, Soura, Srinagar, India. \\ ${ }^{2}$ Department of Radiotherapy, Omega Hospitals, A Unit of Hyderabad Institute of Oncology, Banjara Hills, Hyderabad, India. \\ ${ }^{3}$ Department of Physics, Osmania University, Hyderabad, India. \\ ${ }^{4}$ Biostatistics Division, Sher-i-Kashmir Institute of Medical Sciences, Soura, Srinagar, India.
}

Received May 03, 2014; Revised July 10, 2014; Accepted July 12, 2014; Published Online August 06, 2014

\section{Original Article}

\begin{abstract}
Purpose: The counseling regarding the treatment option is an important objective in the management of early stages breast cancer. The purpose of this study is to present a comparison between the dosimetric aspects of 3DCRT and IMRT in the whole breast radiotherapy. Methods: Both right and left sided computed tomography simulations of 14 women with early stage breast cancer were used for our retrospective study to compare the 3DCRT and IMRT. The dose prescribed was 50 Gray (Gy) in 25 fractions to the whole breast PTV. The PTV was defined by adding unequal margins to the directional safety margin status of each lumpectomy cavity (i.e., medial, lateral, superior, inferior and deep margins measured from the tumor front after the examination of the surgical specimen: 2, 1.5, and $1 \mathrm{~cm}$ for resection margins $<1 \mathrm{~cm}, 1-2 \mathrm{~cm}$, and $>2 \mathrm{~cm}$, respectively) and then modified so that it was no longer closer than $3 \mathrm{~mm}$ to the skin surface and was no deep than the lung -chest interface. The prescribed dose delivered in 5 fractions per week schedule. Treatment plans were compared for target minimum dose, maximum dose, mean dose, conformity index, heterogeneity index and doses to organs at risk were compared and analyzed. Results: The target coverage was achieved with $90 \%$ prescription to the $95 \%$ volume of the PTV. Conformity to the PTV was significantly higher with 3DCRT technique than IMRT. 3DCRT technique seems better in sparing critical organs parameters like lung $\mathrm{V}_{20}$ and Mean, heart, $\mathrm{V}_{25}$, Maximum, both lungs $\mathrm{V}_{20}$, Mean and Dose to the Normal Healthy tissue. Conclusion: We conclude from our study that treatment technique selection for whole Breast irradiation is an important factor in sparing the adjacent normal structures and in determining the associated risk. 3DCRT produces better conformity and heterogeneity indices of the target volume, also reduces dose to OARs and reduces the risk of radiation induced heart diseases.
\end{abstract}

Keywords: Whole Breast Irradiation; Planning Tumor Volume; Organs at Risk; Conformity Index; Heterogeneity Index; Breast Conservative Surgery

\section{Introduction}

An estimated 12.66 million people were diagnosed with cancer across the world in 2008, and 7.56 million people died from the disease. Just four cancer sites lung, female breast, colorectal and stomach accounted for two-fifths of the total cases diagnosed worldwide. Breast cancer is the most common cancer in females worldwide. As per World Health

Corresponding author: Mudasir Ashraf; Department of Radiological Physics and Bio-Engineering, Sher-i-Kashmir Institute of Medical Sciences, Soura, Srinagar, India.

Cite this article as:

Ashraf M, Janardhan N, Bhavani P, Shivakumar R, Ibrahim S, Reddy PY, Surrendharen J, Sarangnathan B, Johnson B, Madhuri $B$, Dar RA. Dosimetric comparison of 3DCRT versus IMRT in whole breast irradiation of early stage breast cancer. Int J Cancer Ther Oncol 2014; 2(3):020318. DOI: 10.14319/ijcto.0203.18
Organization (WHO) estimate, in Bahrain the incidence of breast cancer was 116.47 per 100,000 in 2008 . Unlike other cancers, breast cancer is successfully treatable if detected at an early stage. ${ }^{1}$

The incidence of breast cancer has increased globally over the last several decades. ${ }^{2,3,4}$ The greatest increase has been in Asian countries. ${ }^{5}$ In Asia, breast cancer incidence peaks among women in their forties whereas in the United States and Europe, it peaks among women in their sixties. In India premenopausal patients constitute about $50 \%$ of all patients. ${ }^{6}$ It is expected that in the coming decades, these countries would account for majority of new breast cancer patients 
diagnosed globally. Over 100,000 new breast cancer patients are estimated to be diagnosed annually in India. ${ }^{7}$

Radiotherapy is an integral part of breast cancer management after Breast Conservative Surgery (BCS) in early stage breast cancer. Survival rates are similar for BCS with adjuvant Radiotherapy (RT) and mastectomy for early stage breast cancer and Breast Conservative Surgery (BCS) is known as a gold standard. $8,9,10$ Whole breast irradiation is the commonest method of management for early breast cancer treatment after BCS. The greatest challenge for radiation therapy or any cancer therapy is to attain the highest probability of cure with the least morbidity. The simplest way in theory to increase this therapeutic ratio with radiation is to encompass all cancer cells with sufficient doses of radiation during each fraction, while simultaneously sparing surrounding normal tissues. In practice, however, we have been hampered by our abilities to both identify the cancer cells and target them with radiation. The modern radiotherapy has evolved from non-site-specific techniques using bony anatomy and hand-drawn blocking toward specialized planning incorporating three-dimensional reconstructions of images and computer optimization algorithms. Corresponding to these changes, there has been specialization in the types of technology used for different cancer sites. Two-dimensional (2D) radiotherapy consisted of a single beam from one to four directions. Beam setups were usually quite simple; plans frequently consisted of opposed lateral fields or four field "boxes". Three-dimensional (3D), or Computed Tomography (CT) based, planning was a major advance because it took into account axial anatomy and complex tissue contours and while $3 \mathrm{D}$ planning allows for accurate dose calculations to such irregular shapes, we are still limited in the corrections we could make. The intensity-modulated radiation therapy (IMRT) allows us to modulate the intensity of each radiation beam, so each field may have one or many areas of high intensity radiation and any number of lower intensity areas within the same field, thus allowing for greater control of the dose distribution with the target. By modulating both the number of fields and the intensity of radiation within each field, we have limitless possibilities to sculpt radiation dose. Advanced treatment planning software has furthered our ability to modulate radiation dose. Instead of the clinician choosing every beam angle and weighting, computer optimization techniques can now help determine the distribution of beam intensities across a treatment volume, which often include a non-intuitive distribution of "beamlets," or $1-\mathrm{cm}^{2}$ areas of isointensity. ${ }^{11}$

There are various methods to employ radiotherapy for breast cancer in women. Conventionally tangential fields are employed to treat the whole breast. With the recent advances in treatment planning technology and Multi Leaf Collimators (MLC), Three Dimensional Conformal Radiotherapy (3DCRT) is widely used for treatment of breast carcinoma. Conformal therapy reduces normal tissue doses and increases conformity to target volume. With the advent of advanced sophisticated treatment planning software intensity modulated radiation therapy is becoming increasingly popular. The aim of our study is to compare three dimensional conformal radiation therapy (3DCRT) versus intensity modulated radiation therapy (IMRT) planning based on standard plan indices using Computerized Medical Systems (CMS) Xio (4.70.02 version, ELEKTA) treatment planning system.

\section{Methods and Materials}

\section{CT Imaging}

This study is conducted using treatment plans done on the Computed Tomography (CT) simulation data sets $(2.5 \mathrm{~mm}$ slice thickness) of 14 consecutive patients ( 7 right sided breast carcinoma, 7 left sided breast carcinoma) with $\mathrm{T}_{1}$ or $\mathrm{T}_{2}$ axillary node negative invasive carcinoma of breast for our retrospective analysis. The Planning Tumor Volume (PTV) size varied from $855.73 \mathrm{~cm}^{3}$ to $1347 \mathrm{~cm}^{3}$ with a mean value of $1025.35 \mathrm{~cm}^{3}$. These patients were already treated with IMRT. Radiation Therapy was started within three weeks after the breast conservation surgery consisting of removal of primary tumor with a margin referred as lumpectomy or segmental mastectomy and chemotherapy. A radio opaque wire was placed around the ipsilateral breast by Radiation Oncologist to define the treatment ports for the PTV. The same CT data sets target volumes and organs at risk volumes were used for 3DCRT study.

\section{Target and organ at risk delineation}

After planning CT was done, the Digital Imaging and Communication in Medicine (DICOM) images were transferred to CMS Xio (4.70.02 version, ELEKTA) treatment planning system. The contours that were generated were the Gross Tumor Volume (GTV), Clinical Tumor Volume (CTV), Planning Target Volume (PTV), ipsilateral lung, contralateral lung, contralateral breast, heart, spinal cord and body. The GTV which is the gross tumor volume is the total lumpectomy cavity which can be identified with the help of surgical clips placed at the time of surgery. The CTV was defined by the three dimensional uniform $1.5 \mathrm{~cm}$ margin expanded in all directions around the GTV, however this volume was constrained to lie $5 \mathrm{~mm}$ within the external contour and up against the major muscle. The PTV volume was defined to lie within the radio-opaque wire kept during CT simulation as deep as the anterior chest wall muscles. The lungs and external surfaces contoured using semi-automatic contouring techniques. The CTV, PTV, and Organs at Risk (OARs) were generated in accordance with the Radiation Therapy Oncology Group (RTOG) 0319 protocol. ${ }^{12}$

\section{Treatment Planning Details and Dose Prescription}

All treatment plans were generated with $6 \mathrm{MV}$ (Mega voltage) photon beams to maintain the comparison between the two treatment techniques. Treatment plans were done using 
CMS Xio (4.70.02 version, ELEKTA) for IMRT treatment and 3DCRT treatment.

The treatment fields were almost evenly placed within an arc of 180 degree swept by the gantry. The gantry angles ranged from 300 to 160 (clockwise) for left side tumors and from 60 to 210 (counter clockwise) for right side tumors were chosen to create optimum IMRT plans. The dose prescribed to the Breast volume (PTV) was 50 Grays (Gy) in 25 fractions (2.0 $\mathrm{Gy} /$ fraction). The dose uniformity and conformity were calculated and evaluated.

The Conformity Index (CI) as defined in the International Commission of Radiological Units (ICRU) report 83 is

$$
C I_{(\text {ref })}=\frac{\text { Volume of PTV covered by the reference dose }}{\text { Volume of PTV }}
$$

The Homogeneity Index (HI) as defined in ICRU report 83 is

$$
\begin{gathered}
H I=\frac{D_{5 \%}-D_{95 \%}}{D_{50}} \\
H . I=1 \text { (one) is the ideal value. }
\end{gathered}
$$

Where, $\mathrm{D}_{5 \%}$, $\mathrm{D}_{95} \%$, $\mathrm{D}_{50 \%}$ is the dose received by $5 \%, 95 \%, 50 \%$ volume.

\section{DCRT Planning}

Two opposite tangential beams were constructed to conform to whole breast PTV. A margin of $1 \mathrm{~cm}$ between the MLC and PTV was set for coverage. Using Beam's Eye View (BEV) fields were set up to minimize the dose to heart, left descending coronary artery (LAD), contralateral breasts and ipsilateral lung and maximize the target coverage. The "isocenter" of the treatment machine was positioned at the center of the midline joining the two parallel opposite fields. The plans were created for 3DCRT, in which tangential coplanar beams were used to produce adequate dose coverage for Planning Target Volume (PTV). Critical organs were shielded using MLC without compromising with the PTV coverage. Beam weights were adjusted until the optimum coverage and acceptable hot spots were achieved. The PTV was set to receive $95 \%$ of the prescribed dose. Also, hot spot volumes blocking subfields were determined to achieve the better dose homogeneity and to avoid the overdose in the PTV. By viewing the $105 \%$ dose cloud in a beam's eye view projection of the treatment fields, subfields were designed by blocking the volume of PTV receiving greater than 105\% of the prescribed dose to boost the low dose volume of the PTV (volume of the PTV receiving less the $95 \%$ of the prescribed dose) by delivering 5 to 10 monitor units. The shape of the subfield was iteratively modified with aided visualization of $105 \%$ dose cloud in the beam's eye view. The main field and

\begin{tabular}{|c|c|c|c|}
\hline Organ & Parameter & $\begin{array}{l}\text { Dose } \\
\text { (cGy) }\end{array}$ & Volume (\%) \\
\hline \multirow[t]{2}{*}{ PTV } & $\begin{array}{l}\text { Maximum } \\
\text { Dose }\end{array}$ & 5500 & 0 \\
\hline & $\begin{array}{l}\text { Minimum } \\
\text { Dose }\end{array}$ & 4700 & 100 \\
\hline Ipsilateral Lung & Mean Dose & 2000 & 30 \\
\hline $\begin{array}{l}\text { Contralateral } \\
\text { Lung }\end{array}$ & Mean Dose & 2000 & $\begin{array}{l}\text { 33\% volume of } \\
\text { (Lung-PTV) }\end{array}$ \\
\hline $\begin{array}{l}\text { Contralateral } \\
\text { Breast }\end{array}$ & Mean Dose & 1500 & $5 \%$ \\
\hline \multirow[t]{2}{*}{ Heart } & Mean Dose & 3500 & 0 \\
\hline & Point Dose & 500 & $\begin{array}{l}\text { Less than the Pre- } \\
\text { scribed dose }\end{array}$ \\
\hline
\end{tabular}
subfields were merged into one portal.

\section{IMRT Planning}

TABLE 1: Dose constraints for 3DCRT and IMRT planning.

In this technique, the fluence based step and shoot IMRT optimized plans were generated to achieve the same objectives described for the 3DCRT plans, the number of beam segments was not restricted during optimization and no attempts have been made for beam angle optimization as CMS Xio does not support beam angle optimization. The PTV for IMRT was the same as used for the 3DCRT plans plus an extension into the air anterior of the PTV of $1.5 \mathrm{~cm}$ in order to compensate the set up uncertainties and up and down movement of the chest due to respiration. The dose was prescribed to the PTV and the dose constraints presented in the Table 1 were set to the treatment planning system. Tissue heterogeneity was considered in the treatment planning optimization process and the dose calculation was done by using superposition/ convolution algorithm.

The CMS Xio (4.70.02 version, ELEKTA) treatment planning system Dose Volume Histogram (DVH) window was used to analyze the PTV's mean, maximum and minimum doses and for OAR's mean, maximum, minimum doses, percentage of volume receiving 3000cGy (V30), percentage of volume receiving 2000 cGy ( $\left.\mathrm{V}_{20}\right)$, percentage of volume receiving 1000 cGy $\left(\mathrm{V}_{10}\right)$, and isodose volumes for $50 \%, 30 \%$ and $10 \%$. Also to illustrate the low dose volume effects, Dose Volume Histogram for normal healthy tissue are also incorporated and Monitor Units (MU) were also noted in order to access and understand integral dose (ID) contribution, the Monitor Units (MU) for both the plans were noted and taken for analysis. The more monitor unit signifies the more integral dose. The integral dose ID to an organ $\mathrm{j}$ divided into $\mathrm{m}$ voxels is given by the following equation: ${ }^{3}$

$$
I D=\sum_{k=1}^{m} V_{k j} D_{k j} \rho_{k l}
$$

Where, $V_{k j}$, $D_{K J}$ and $P_{k l}$ are respectively the volume, dose and density of voxel $\mathrm{k}$ in organ $\mathrm{j}$. If the voxels have all the same size and the organ can be assumed to have a uniform density, the integral dose equation can be reduced to $I D=V_{j} \rho_{j} D_{j}$ 


\section{Statistical Analysis}

For qualitative analysis of the two techniques, a very suitable method of investigation is provided by Wilcxon's Signed Rank sum test, which operates as follows. First, put all the observations in ascending order of their magnitude, ignoring the signs. Any zero values are ignored and the remaining non-zero values are assigned rank 1 to $n$. If any of the observations are numerically equal they are each assigned an average rank calculated from the rank that would otherwise have been used. Such ranks are said to be tied. Next calculate the sum of the ranks of the positive observations and refer the table for appropriate significance level. ${ }^{14}$

The above said statistical analysis was performed for qualitative ranking of the two techniques using Statistical Package for Social Sciences (SPSS) version12.0 (SPSS Inc., Chicago, USA) software. This matched pair t-test was applied to determine the statistical difference between the dose volume data for IMRT versus 3DCRT. The reported p-value is two tailed and p-values of $<0.05$ are considered significant or else non-significant (NS).

\section{Results}

TABLE 2: (a) Comparison of average dosimetric characteristics for left Whole Breast PTV for IMRT and 3DCRT technique. (Prescribed dose 5,000 cGy in 25 fractions).

\begin{tabular}{lccc}
\hline \hline Dosimetric Parameter & IMRT & 3DCRT & p-Value \\
\hline Max Dose (cGy) & 5594.34 & 5606.43 & $0.055^{*}$ \\
Min Dose (cGy) & 2249.47 & 1714.17 & 0.039 \\
Mean Dose (cGy) & 4984.34 & 5041.63 & 0.031 \\
95\% volume & 4567.4 & 4565.24 & $0.063^{*}$ \\
Conformity Index & 0.90 & 0.89 & $0.063^{*}$ \\
Heterogeneity Index & 1.16 & 1.14 & 0.023 \\
Monitor Unit (MU) & 761.4 & 260.6 & 0.008 \\
\hline \hline
\end{tabular}

$*$ statistically insignificant

TABLE 2: (b) Comparison of average dosimetric characteristics for right Whole Breast PTV for IMRT and 3DCRT technique. (Prescribed dose 5,000 cGy in 25 fractions).

\begin{tabular}{llll}
\hline \hline Dosimetric Parameter & IMRT & 3DCRT & p-Value \\
\hline Max Dose (cGy) & 5618.96 & 5565.96 & $0.063^{*}$ \\
Min Dose (cGy) & 2842.13 & 1171.70 & 0.008 \\
Mean Dose (cGy) & 4945.33 & 5172.94 & 0.039 \\
95\% volume & 4537.56 & 4681.44 & 0.016 \\
Conformity Index & 0.94 & 0.95 & $0.125^{*}$ \\
Heterogeneity Index & 1.12 & 1.10 & 0.024 \\
Monitor Unit (MU) & 2502 & 1297.4 & 0.008 \\
\hline \hline
\end{tabular}

${ }^{*}=$ statistically insignificant

The treatment plans were optimized to meet the planning objectives in both the treatment techniques and to achieve the prescribed dose delivery for more than $90 \%$ of the prescribed isodose to encompass greater than $95 \%$ of the PTV volume. The PTV size varied from $855.73 \mathrm{~cm}^{3}$ to $1347 \mathrm{~cm}^{3}$ with a mean value of $1025 \pm 35 \mathrm{~cm}^{3}$. In 3DCRT, $105 \%$ dose (hot spots) was observed in less than $5 \%$ of the target volume. The normalized target coverage for IMRT and 3DCRT in PTV and for both classes of the patients were presented in the Table 2a and $2 \mathrm{~b}$. Figure 1 demonstrates the dose distribution in axial sections for the two classes of patients (right sided diseases patient and left sided disease patient) for IMRT and 3DCRT plans of the same patient. The axial sections clearly depict the concave target PTV coverage and in all left sided breast patients, the left anterior descending coronary artery (LAD) is little away from the PTV and not receiving any significant dose in both the techniques.

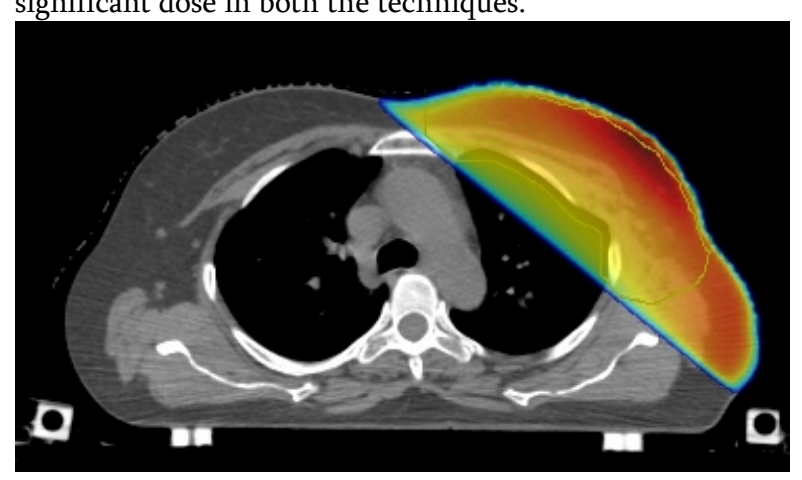

(a)

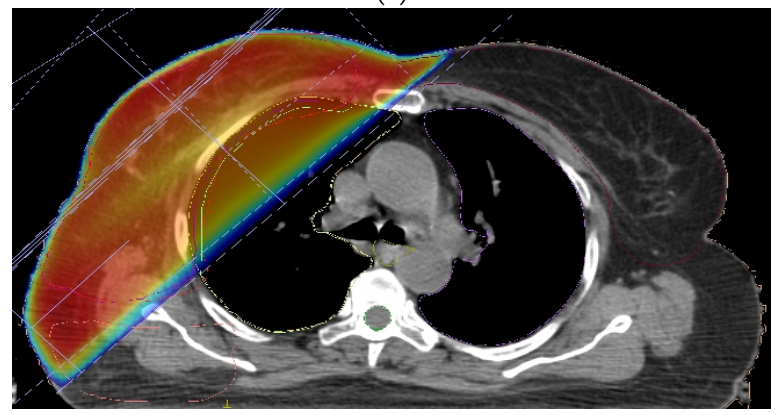

(b)

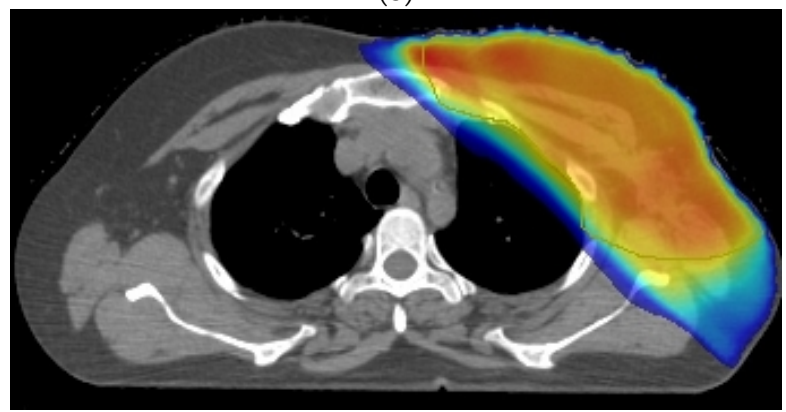

(c)

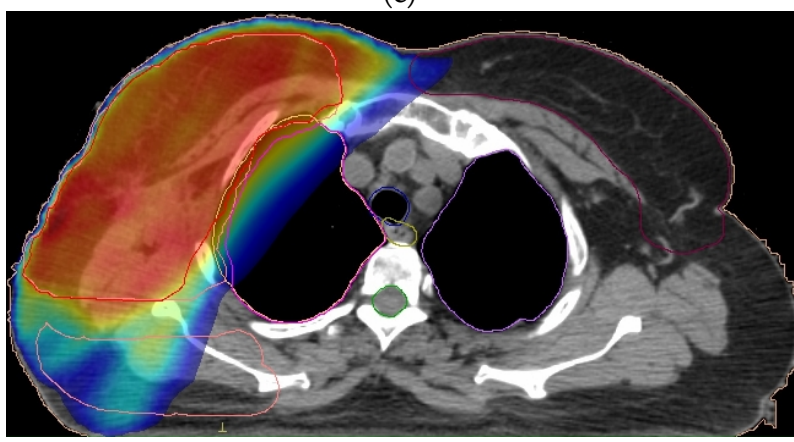

(d)

FIG. 1: Represents the 20 Gy volume of dose color wash in 3DCRT (a, b) and IMRT (c, d) breast plans. 
The comparison and dosimetric analysis for the Planning Target volume (PTV) presented in the Table $2 \mathrm{a}, \mathbf{2 b}$ depicts that there is no significant difference in maximum dose and conformity index in both the treatment techniques. However, the heterogeneity index was found to be 1.16 for IMRT and 1.14 for 3DCRT $(\mathrm{p}=0.023)$ in left breasts and 1.12 for IMRT and 1.10 for 3DCRT ( $\mathrm{p}=0.024)$ in the right breasts. All the dosimetric parameter calculated for the Planning Target Volume (PTV) except mean dose has appreciably higher values in IMRT plans than 3DCRT plans. Moreover, the dosimetric analysis carried out for ipsilateral lung dose parameters like mean dose, $\mathrm{V}_{5}, \mathrm{~V}_{10}$ were found to have higher values in all IMRT plans than 3DCRT plans. The difference is presented in Table 3 for both classes of the patients.

TABLE 3: Comparison of mean values for ipsilateral lung parameters for IMRT and 3DCRT in left sided breasts patients.

\begin{tabular}{lllll}
\hline \hline Dosimetric Parametric & IMRT & 3DCRT & p-value \\
\hline$V_{5}(\%)$ & 57.70 & 24.76 & 0.008 \\
$V_{20}(\%)$ & 34.0 & 41.00 & 0.039 \\
$V_{30}(\%)$ & 26.94 & 32.65 & 0.031 \\
$V_{10}(\%)$ & 57.51 & 28.97 & 0.008 \\
Mean Dose (cGy) & 1377.96 & 1065 & 0.023 \\
\hline \hline
\end{tabular}

Figure 6 illustrates the Dose Volume Histogram (DVH) for heart. A comparison of dosimetric parameters for heart is presented in Table $4 \mathrm{a}, 4 \mathrm{~b}$. These parameters show much higher values in IMRT technique than 3DCRT technique except the dose value to the $33 \%$ of the heart volume and the volume encompassed by $25 \%$ isodose line.

TABLE 4: (a) Comparison of mean values for heart dose parameters for IMRT and 3DCRT in right sided breasts patients.

\begin{tabular}{llll}
\hline \hline Dosimetric Parameter & IMRT & 3DCRT & p-value \\
\hline Max Dose (cGy) & 3308.41 & 2315.05 & 0.039 \\
Min Dose (cGy) & 259.77 & 17.96 & 0.008 \\
Mean Dose (cGy) & 1198.1 & 149.84 & 0.047 \\
$33 \%$ Volume (cGy) & 710.5 & 124.56 & 0.008 \\
V25 (\%) & 2.34 & 1.80 & $0.055^{*}$ \\
V10 (\%) & 20.72 & 2.95 & 0.016 \\
\hline \hline
\end{tabular}

${ }^{*}=$ statistically insignificant

TABLE 4: (b) Comparison of mean values for heart dose parameters for IMRT and 3DCRT in left sided breasts patients.

\begin{tabular}{llll}
\hline \hline Dosimetric Parameter & IMRT & 3DCRT & p-value \\
\hline Max Dose (cGy) & 4699.99 & 4515.53 & 0.039 \\
Min Dose (cGy) & 330.30 & 50.57 & 0.008 \\
Mean Dose (cGy) & 1499.57 & 1184.24 & 0.008 \\
$33 \%$ Volume (cGy) & 1035.84 & 1465.79 & $0.055^{*}$ \\
V $_{25}(\%)$ & 16.02 & 20.66 & $0.063^{*}$ \\
$V_{10}(\%)$ & 47.73 & 27.06 & 0.016 \\
\hline \hline
\end{tabular}

*= statistically insignificant

A comparison of average plan parameters for contralateral breasts and both lungs is presented in Tables 5, and 6 respectively. Dose Volume Histograms (DVHs) describing the dose volume relation of the target as well as normal tissue for both the techniques is presented in Figure 2-7. The mean value of Monitor Units (MU) for left sided diseases in IMRT technique was found to be 761.4 Monitor Units (MUs) as compared to 260.0 Monitor Units (MUs) in 3DCRT with ( $\mathrm{p}=0.008$ ) and for right sided diseases the average value of Monitor Units in IMRT technique was observed to be 2502 Monitor Units (MUs) compared to 1297.4 monitor units (MUs) in 3DCRT $(\mathrm{p}=0.008)$. The value of the monitor unit will depend on the depth that we are going to treat. The higher value of MUs in case of right sided breast disease may only because of large breast volume or because of the pendulous breasts. Also, the DVH shows considerably better normal tissue sparing with 3DCRT than IMRT for the intact whole breasts.

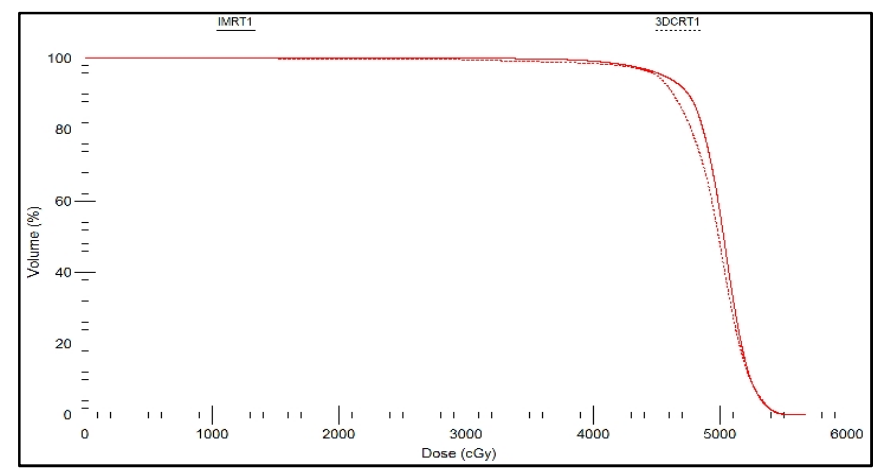

(a) DVH of PTV in IMRT and 3DCRT plans for right breast cancer case

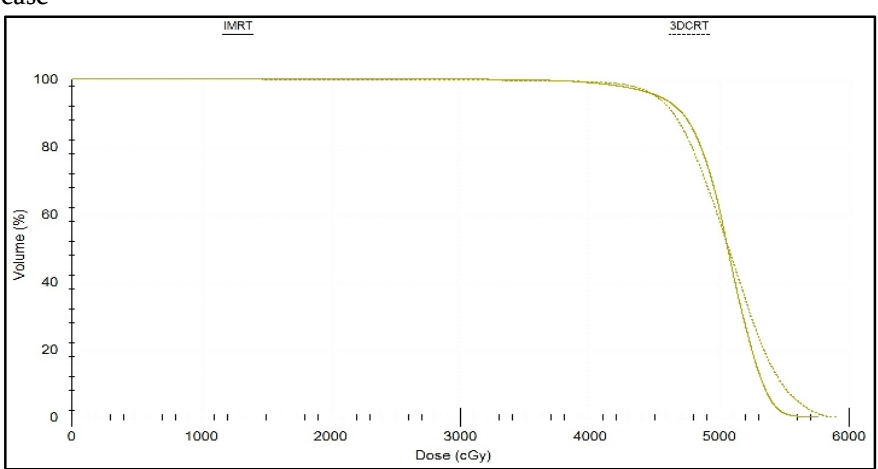

(b) DVH of PTV in IMRT and 3DCRT plans for left breast cancer case FIG. 2: Cumulative dose volume histogram (DVH) of PTV comparing 3DCRT and IMRT plans for (a) right breast, and (b) left breast cancer cases.

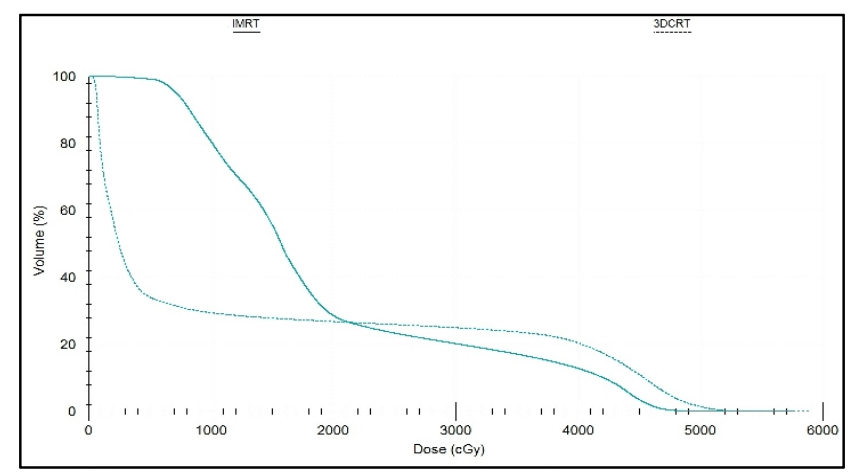

FIG. 3: Cumulative dose volume histogram (DVH) of ipsilateral lung comparing 3DCRT and IMRT plans. 


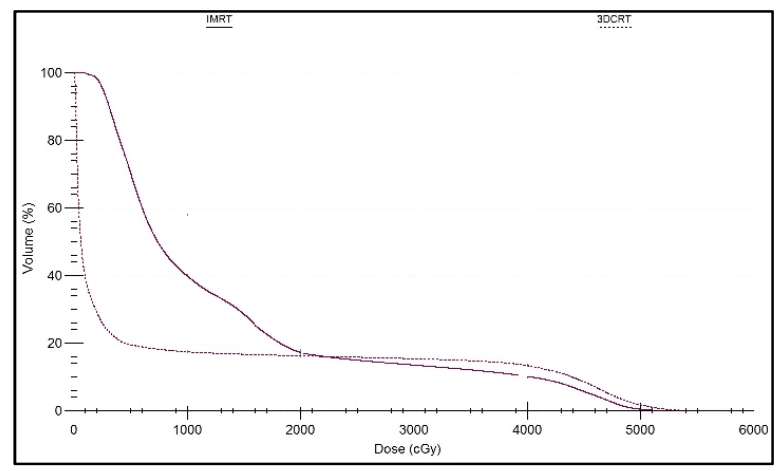

FIG. 4: Cumulative dose volume histogram of both lungs comparing 3DCRT and IMRT plans.

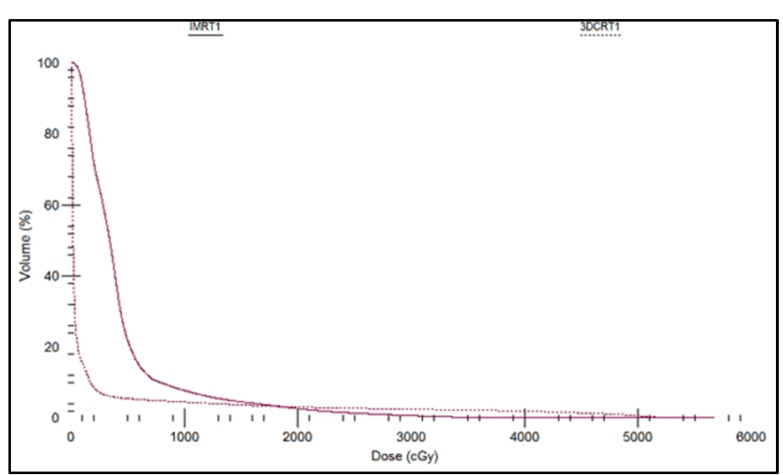

FIG. 5: Cumulative dose volume histogram of contralateral breast comparing 3DCRT and IMRT plans.

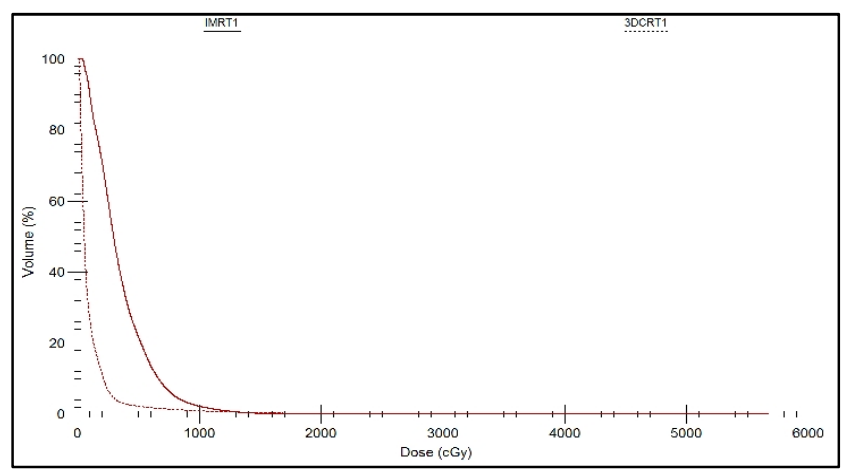

(a)DVH of heart in IMRT and 3DCRT plans for right breast cancer case

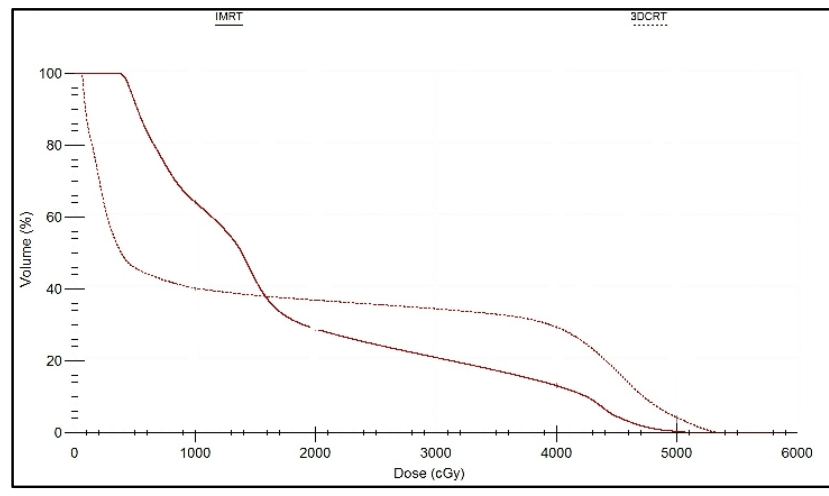

(b) DVH of heart in IMRT and 3DCRT plans for left breast cancer case. FIG. 6: Cumulative dose volume histogram of heart comparing 3DCRT and IMRT plans for (a) right breast cancer, and (b) left breast cancer cases

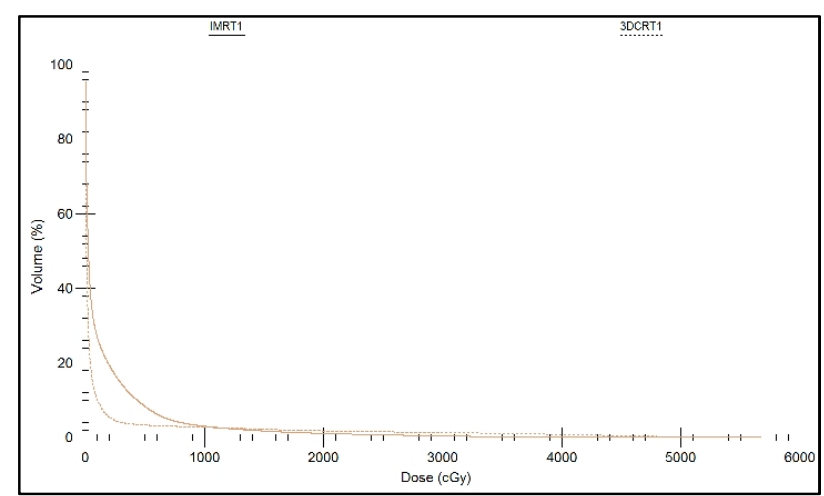

(a) DVH of normal tissue in IMRT and 3DCRT plans for right breast

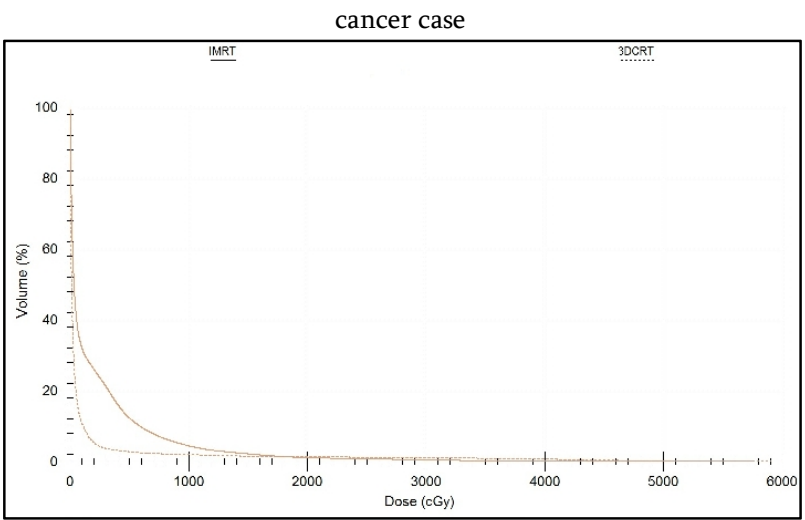

(b)DVH of normal tissue in IMRT and 3DCRT plans for left breast cancer case

FIG. 7: Cumulative dose volume histogram (DVH) of normal healthy tissue comparing 3DCRT and IMRT plans for (a) right breast cancer, and (b) left breast cancer cases.

TABLE 5: Comparison of mean values for contralateral Breast dose parameters for IMRT and 3DCRT in right sided breasts patients.

\begin{tabular}{lllll}
\hline \hline Dosimetric parameter & IMRT & 3DCRT & p-value \\
\hline V5 $(\%)$ & 12.065 & 8.30 & 0.031 \\
Min Dose (cGy) & 188.92 & 181.63 & 0.039 \\
D5 (\%) & 737.38 & 786.2 & 0.027 \\
Max Dose (cGy) & 3861.00 & 4755.55 & 0.008 \\
Mean Dose (cGy) & 269.71 & 226.20 & 0.039 \\
\hline \hline
\end{tabular}

TABLE 6: Comparison of mean values for both lungs dose parameters for IMRT and 3DCRT in left sided breasts patients.

\begin{tabular}{llll}
\hline \hline Dosimetric Parametric & IMRT & 3DCRT & p-value \\
\hline$V_{20}(\%)$ & 20.45 & 17.00 & 0.016 \\
Mean Dose (cGy) & 1892.17 & 778.69 & 0.016 \\
\hline \hline
\end{tabular}

\section{Discussion}

Several epidemiological studies have shown that in country like India breast cancer patients usually come in advanced stage of breast cancer, breast conservative surgery is not possible, so mastectomy is the treatment of choice and early stage breast cancer is rare. In early stage breast cancer, breast conservative surgery is known as gold standard. There are distinct geometric differences between the target volume of the chest wall and the whole breast, and these differences might have an impact on the resulting dose distribution. In general, 
there is an optimum plan for every patient that treats the breast tissue while sparing the organs at risk. However, the technique one may use could vary depending on the patient geometry and technology available in a particular radiotherapy center. The number of studies has been carried out to present the superiority of one technique over other. A very recent study by Suresh Moorthy et al. ${ }^{15}$ presents a Dosimetric study of SIB-IMRT versus SIB-3DCRT for breast cancer with breath-hold gated technique. The study envisages that in comparison to 3DCRT, IMRT reduced the maximum dose to the target volume, and dose to OAR was reduced too.

However, 3DCRT technique was superior in terms of low dose volume of normal tissue, integral dose, and treatment time. Consequences of these low doses would have to be weighed against the benefits of reducing high doses on individual patient selection basis. With the use of breath-hold gated technique in IMRT, it can further improve the target coverage and reduction of doses to the heart, lung, and LAD. SIB technique could reduce the overall treatment duration by about one week. The present study is intended to compare the planning and dose delivery efficiency among two treatment techniques of radiation therapy to the whole breast namely three dimensional Conformal Radiotherapy (3DCRT) and Intensity Modulated Radiotherapy (IMRT) in fair manner for post lumpectomy breast cancer patients with CMS Xio (4.70.02 version, ELEKTA) treatment planning system.

In this study tangential beam 3DCRT of the whole breast PTV is compared to IMRT. Table 2 depicts that the IMRT results are comparable to the 3DCRT technique in terms of the PTV coverage, delivering $90 \%$ of the prescribed dose to greater than $95 \%$ volume of the PTV, maximum dose delivered to the whole breast PTV, and conformity index. The Monitor Units in IMRT technique was higher than 3DCRT technique with $p$ - values 0.008 . This envisages that the integral dose would be higher probably due to multiple beams used in the IMRT plans than tangentially oriented beams used in three dimensional conformal plans to spare adjacent normal healthy tissue structures. The leakage and scatter dose to the non-target tissue is proportional to the number of Monitor Units used. Some studies reported, increased low dose volumes with increased number of beam angles increase the integral dose. ${ }^{16}$

In general, both IMRT and 3DCRT provided similar results regarding the PTV coverage. But, in depth analysis of dosimetric data reveals significant difference in the quality of the target coverage and normal tissue doses. For tangential beam 3DCRT technique the heterogeneity index for both left and right sided breasts disease was nearer to the ideal value compared to IMRT technique. While as, conformity indices were found more or less equal. The 3DCRT technique reduces the values for lung dose volume, heart dose volume, dose to contralateral breast and dose to both lungs than IMRT technique without compromising the target coverage. In this study, all most all the ipsilateral lung dose parameters were observed to have higher values in IMRT technique than 3DCRT technique. The Radiation Induced Pneumonitis is related to the ipsilateral lung volume irradiated, ${ }^{17}$ the ipsilateral lung $\mathrm{V}_{20}$ for IMRT (34.0\%) is significantly less than $(41.00 \%)$ in 3DCRT $(p=0.039)$. Both lungs $V_{20}$ parameters have significantly lower values in 3DCRT technique than IMRT with $p$-values 0.016 . There is no absolute safe Mean Lung Dose (MLD) below which there no Pneumonitis. ${ }^{18}$ The clinically acceptable risk of radiation therapy depends on the risk benefit ratio of the individual patient selection.

In patients with left sided breast cancer, it is intended that the irradiated heart volume be minimized to the greatest possible degree without compromising the target coverage as the risk of pericardial events is probably related to both dose and volume of irradiation. Stewart J R et al. ${ }^{19}$ concludes that the dose volume should be limited to $60 \mathrm{~Gy}$ for less than $20 \%$ of the cardiac volume and $45 \mathrm{~Gy}$ for more than $65 \%$ of cardiac volume. A very recent study on "Risk of Ischemic Heart Diseases in Women after Radiotherapy for Breast Cancer" by Sarah CD et al. ${ }^{20}$ suggests that the exposure of heart to ionizing radiations during radiotherapy for breast cancer increases the subsequent rate of ischemic heart diseases. The increase is proportional to the mean dose to heart, begins within few years after exposure, and continues at least for 20 years. Woman with preexisting cardiac risk factors have greater absolute increase in risk from radiotherapy than other women. Also, the rate of major coronary events increased linearly with the mean dose to the heart by $7.4 \%$ per Gray, with no apparent threshold. The risk starts within 5 years after radiotherapy and continuous up to the third decade after radiotherapy.

The IMRT plans contribute a modestly higher dose to adjacent healthy tissues. The main concern of with the healthy soft tissue dose increases of such magnitude is an increased risk of late secondary malignancy. ${ }^{21,22}$

\section{Conclusion}

The objective of this study was to compare the dosimetric characteristics of IMRT and 3DCRT techniques and to evaluate the characteristics of each modality when applied to the whole breast radiotherapy in the early stage of the breast cancer. The quality of the treatment plan dependence on the many factors, in general, there is an optimum plan for every patient that treats the breast tissue while sparing the organs at risk. However, the technique one may use could vary depending on the patient geometry and technology available in a radiotherapy center such as treatment planning system available, beam energy, TPS algorithm and the skills of the planner as shown by $\mathrm{Lu}^{23}$, Rana ${ }^{24}$, and Pokharel. ${ }^{25}$

IMRT is now considered to be a mature radiotherapy technique and has become a frequently used modality in all most 
all the radiotherapy centers. The MLC-based IMRT technique delivers non-uniform fluences to the patient from any given position of the treatment beam to optimize the composite dose distribution and to spare the adjacent organs at risk.

We infer from this study that treatment technique selection for whole-breast irradiation is an important factor in sparing the adjacent normal structures and in determining the associated risk. 3DCRT reduces the dose to the OARs and increases the heterogeneity index on CMS Xio (4.70.02 version, ELEKTA) treatment planning system. Quantification of dose to OARs may be useful for clinicians as they counsel women with early stage breast cancer about their treatment option Moreover, according to the Sarah CD et al. ${ }^{19}$ recent study on "Risk of Ischemic Heart Diseases in Women after Radiotherapy for Breast Cancer", the 3DCRT reduces the risk of radiation induced heart diseases by a factor of about 9.62 in right sided breast diseases than IMRT and by a factor of 1.27 in left sided breast diseases.

\section{Conflict of interest}

The authors declare that they have no conflicts of interest. The authors alone are responsible for the content and writing of the paper.

\section{Acknowledgment}

We are highly thankful to Professor (Dr.) Mohan Vamsy, Managing Director and Chief Surgical Oncologist, Omega Hospitals, A Unit of Hyderabad Institute of Oncology for allowing us to carry out the study in his esteemed institute. The authors would also like to thank Ayyangar Komandari, former professor at the University of Nebraska, USA for his valuable discussions and suggestions pertaining to this work.

\section{References}

1. Cancer Stats. Cancer Worldwide, International agency for research on Cancer. World Health Organization (WHO); 2011.

2. Khokhar A. Breast cancer in India: where do we stand and where do we go? Asian Pacific J Cancer Prev 2012; 13: 4861-6.

3. Hortobagyi GN, de la Garza Salazar J, Pritchard K, et al. The global breast cancer burden: variations in epidemiology and survival. Clin Breast Cancer 2005; 6: 391-401.

4. Anderson BO, Jakesz R. Breast cancer issues in developing countries: an overview of the breast health global initiative. World J Surg 2008; 32: 2579-85.

5. Green M, Raina V. Epidemiology, screening and diagnosis of breast cancer in the Asia-Pacific re- gion: current perspectives and important considerations. Asia-Pacific Journal of Clinical Oncology 2008; 4: 5-13.

6. Agarwal G, Pradeep PV, Aggarwal V, et al. Spectrum of breast cancer in Asian women. World $J$ Surg 2007; 31:1031-40.

7. Nandakumar A, Anantha N, Venugopal TC, et al. Survivalin breast cancer: a population-based study in Bangalore, India Int J Cancer 1995; 60:593-6.

8. Kutcher GJ, Smith AR, Fowble BL, et al. Treatment planning for breast cancer: a patterns of care study. Int J Radiat Oncol Biol Phys 1996; 36:731-7.

9. Holland R, Veling SH, Mravunac M, Hendriks JH. Histologic multifocality of Tis, T1-2 breast carcinomas: implications for clinical trials of breast conserving surgery. Cancer 1985; 56: 979-90.

10. Ercan T, İgdem S, Alco G, et al. Dosimetric comparison of field in field intensity-modulated radiotherapy technique with conformal radiotherapy techniques in breast cancer. Jpn J Radiol 2010; 28:283-9.

11. Bucci MK, Bevan A, Roach M $3^{\text {rd }}$. Advances in radiation therapy: conventional to $3 \mathrm{D}$, to IMRT, to 4D, and beyond. CA Cancer J Clin 2005; 55:117-34.

12. D'Arienzo M, Masciullo SG, de Sanctis V, et al. Integral dose and radiation-induced secondary malignancies: comparison between stereotactic body radiation therapy and three-dimensional conformal radiotherapy. Int J Environ Res Public Health 2012; 9:4223-40.

13. Vicini F, Winter K, Straube W, et al. A phase I/I trial to evaluate $3 \mathrm{D}$ conformal radiation therapy confined to the region of the lumpectomy cavity for stage I/II breast carcinoma: initial report of feasibility of and reproducibility of radiation therapy oncology group (RTOG) 0319. Int J Radiat Oncol Biol Phys 2005; 63: 1531-7.

14. Richard F Mould. Introductory Medical Statistics, 3rd edition, IOP Publishing Ltd, chapter 13. 1998

15. Moorthy S, Sakr H, Hasan S, Samuel J, et al. Dosimetric study of SIB-IMRT versus SIB-3DCRT for breast cancer with breath-hold gated technique. Int J Cancer Ther Oncol 2013; 1:010110.

16. Cho BC, Schwarz M, Mijnheer BJ, Bartelink H. Simplified intensity modulated radiotherapy using pre-defined segments to reduce cardiac complications in left-sided breast cancer. Radiother Oncol 2004; 70:231-41.

17. Recht A1, Ancukiewicz M, Alm El-Din MA, et al. Lung dose-volume parameters and the risk of pneumonitis for patients treated with accelerated partial-breast irradiation using three-dimensional conformal radiotherapy. J Clin Oncol 2009; 27:3887-9. 
18. Marks LB, Bentzen SM, Deasy JO, et al. Radiation dose-volume effects in the lung. Int J Radiat Oncol Biol Phys 2010; 76: S70-6.

19. Stewart JR, Gajardo LF, Gillette SM, Constine LS. Radiation injury to the heart. Int J Radiat Oncol Biol Phys 1995; 31:1205-12.

20. Darby SC, Ewertz M, McGale P, et al. Risk of ischemic heart disease in women after radiotherapy for breast cancer. NEng J Med 2013; 368: 987-98.

21. Gagliardi G, Constine LS, Moiseenko V, et al. Radiation dose-volume effects in the heart. Int J Radiat Oncol Biol Phys 2010; 76: S77-85.

22. Rongsriyam K, Rojpornpradit P, Lertbutsayanukul $\mathrm{C}$, et al. Dosimetric study of inverse-planed intensity modulated, forward-planned intensity modulated and conventional tangential techniques in breast conserving radiotherapy. J Med Assoc Thai 2008; 91: 1571-82.
23. Lu L. Dose calculation algorithms in external photon beam radiation therapy. Int J Cancer Ther Oncol 2013; 1: 01025.

24. Rana S. Clinical dosimetric impact of Acuros XB and analytical anisotropic algorithm (AAA) on real lung cancer treatment plans: review. Int J Cancer Ther Oncol 2014; 2:02019.

25. Pokharel S. Dosimetric impact of mixed-energy volumetric modulated arc therapy plans for high risk prostate cancer. Int J Cancer Ther Oncol 2013; 1: 01011. 